CAHIERS DE

NARRATOLOGIE

\section{Cahiers de Narratologie}

Analyse et théorie narratives

$28 \mid 2015$

Le récit comme acte cognitif

\title{
Dynamique narrative du texte, du film et de la musique
}

\section{Wolfgang Wildgen}

\section{(2) OpenEdition}

\section{Journals}

Electronic version

URL: https://journals.openedition.org/narratologie/7243

DOI: $10.4000 /$ narratologie. 7243

ISSN: 1765-307X

Publisher

LIRCES

\section{Electronic reference}

Wolfgang Wildgen, "Dynamique narrative du texte, du film et de la musique", Cahiers de Narratologie [Online], 28 | 2015, Online since 03 November 2015, connection on 11 June 2021. URL: http:// journals.openedition.org/narratologie/7243 ; DOI: https://doi.org/10.4000/narratologie.7243

This text was automatically generated on 11 June 2021.

Article L.111-1 du Code de la propriété intellectuelle. 


\title{
Dynamique narrative du texte, $\mathrm{du}$ film et de la musique
}

\author{
Wolfgang Wildgen
}

\section{Introduction}

Le discours narratif peut être organisé dans des modes très différents. Il peut être oral ou écrit dans le cas du récit et du texte, il peut être visuel comme le film muet ou essentiellement visuel, mais soutenu et élaboré par la parole, les bruits et la musique dans le film moderne. Enfin il peut être mimique, gestuel ou dansé (dans la pantomime et le ballet). Il est difficile de s'imaginer un récit olfactif ou gustatif ; pourtant une suite de repas ou de vins dégustés pourrait à la limite constituer un récit. Quel est donc la condition pour qu'une suite des manifestations symboliques soit un récit, une gestalt narrative? D'abord il y a le temps, le changement. Contrairement à un tableau descriptif, par exemple une nature morte, le récit demande une suite dans le temps, un avant et un après, un début et une fin, etc. On pourrait objecter que le fleuve qui coule, les vagues qui déferlent, les nuages qui passent (peints ou filmés) sont aussi dans le temps, mais il leur manque un élément décisif qui caractérise le récit : c'est la coupure (catastrophiste) ou la transition (abrupte) d'une situation à une autre. Il faut ajouter que cette transition, qui n'est ni lisse, ni continue, ni imperceptible, renvoie à des forces. Ceci introduit la dynamique qui depuis Newton et Kant est le domaine des forces qui décident de la direction et vitesse du mouvement. En général l'homme a tendance à voir les forces comme signes d'une intention, d'une volonté, donc dans le contexte d'un acte humain. Si cette volonté reste cachée, on imagine des esprits, démons, dieux, etc. Si nous parlons de «dynamique narrative ", nous mettons en relief cet aspect du récit tout en cherchant une connexion avec les disciplines qui ont développé l'étude de la dynamique depuis Newton (la physique, la chimie, la biologie) ou qui ont introduit les moyens formels pour décrire cette dynamique : le calcul différentiel, la topologie, et en général la théorie des systèmes dynamiques, dont la théorie des catastrophes, à la quelle nous ferons référence à plusieurs reprises.

L'analyse cognitive d'un système de signes et de son utilisation connaît plusieurs niveaux. D'abord il y a le niveau fondamental des mécanismes sensoriels et moteurs (1), le hard-ware corporel, qui est inné et spécifique pour l'espèce Homo sapiens ; puis il y a 
le niveau de l'adaptation de ces mécanismes au contexte vital (2), les champs de perception (lumière, sons, odeurs, etc.) et de motricité (physique du corps propre et des objets auxquels il touche, qu'il manipule). Enfin il y a le niveau de la culture ambiante qui forme le cerveau par voie de l'apprentissage et de la réflexion (3). Si nous considérons le récit langagier (le texte oral et écrit), l'image, le film et la musique, nous pouvons observer immédiatement qu'au niveau fondamental (1) ils sont différents. L'image renvoie au visuel et dans sa production à la motricité de la main, la musique renvoie à l'ouie et aux articulateurs phonétiques, dans le cas des instruments musicaux à la motricité de la bouche, des mains, etc. Les systèmes sensoriels (l'œil, l'oreille) sont nettement différents et ceci vaut aussi pour les réseaux neuronaux à un niveau primordial. Il y a pourtant des réseaux secondaires et tertiaires qui sont moins localisées et se recoupent. Le cas du texte linguistique est plus compliqué, car il apparaît d'abord sous forme orale, donc se rapportant à l'ouïe et à la bouche, et après à l'écriture, donc à la vision et à la main qui écrit. Le fait que la vision et l'ouĩe soient plus fondamentales renvoie à une succession évolutionnaire : le langage est beaucoup plus récent que notre capacité à percevoir et à écouter. La succession dans la maturation corporelle donne la priorité à l'ouie (avant la naissance), à la vue (dès la naissance) ; puis, avec beaucoup de retard, le langage apparait. Considéré à ce niveau fondamental, le «texte» musical serait prioritaire, suive du «texte» visualisé et enfin du texte langagier. Au deuxième niveau, la référence au contexte, la prise en considération du monde externe, la musique et la vision sont aussi prioritaires, car elles établissent un lien direct aux fréquences des vibrations de l'air, des distances et même de l'emplacement de la source du bruit, du son dans le cas de l'ouïe et au lieu d'un objet perçu, de sa forme/taille et de ses caractéristiques (contours, couleurs, etc.), dans le cas de la vision. Le langage au contraire présente une ontologie mixte, aussi bien dans sa forme (acoustique, visuelle) que dans son contenu, qui renvoie à tous les types de perceptions et même à des entités imaginaires. Sous l'aspect neuronal cela veut dire que le langage dans sa forme symbolique (non seulement phonétique ou graphique) renvoie à une multitude de processus neuronaux non localisés, distribués. Le niveau culturel (3) rapproche pourtant les différents modes, c'est-à-dire que leur correspondance et en conséquence la possibilité d'une interaction, d'un mélange (calque) apparaît à ce niveau. Les "représentations collectives » (selon Durkheim) ont certainement une réalité neuronale, mais celle-ci n'a aucune raison d'être identique chez les individus. Au contraire, les processus d'apprentissage varient avec les contextes et la gestalt neuronale de la culture chez l'individu est imprévisible. Il ne vaut pas la peine de chercher la culture humaine dans le cerveau à l'aide des techniques modernes de visualisation, car la "mémoire collective" laisse des traces très différentes dans les cerveaux individuels, sans pourtant annihiler le phénomène social et culturel. C'est au niveau culturel (3) que le mode linguistique domine; les modes visuels et musicaux héritent les moyens de déployer la forme symbolique en rivalité avec la complexité culturelle du langage. Comme le discours narratif fait partie de cette dynamique complexe, il est naturel que la modalité dominante du narratif soit linguistique, et je vais commencer par elle mon parcours analytique et argumentatif.

Morphogenèse du récit spontané et d'une tradition narrative ${ }^{1}$

Le texte narratif est toujours le produit d'une situation narrative, de l'activité d'un narrateur. La situation normale et prototypique de la production d'un texte narratif est la situation 'face à face' et la production orale. Le contenu du récit est ou bien un vécu personnel (1), ou bien le contenu d'une narration précédente $(2)^{2}$, ou encore un 
héritage culturel qui renvoie à un univers de discours hors de notre horizon personnel. Ceci se vérifie dans les contes populaires (3). Ces contes peuvent faire l'objet d'une rédaction littéraire, donc d'un type spécial de reproduction (4). Nous allons surtout traiter les trois premiers types de textes dans l'ordre indiqué. Cet ordre implique une échelle diachronique comprenant une rupture temporelle en son milieu. Nous choisirons deux phases bien distinctes : (1) et (3).

La dynamique du récit oral et spontané

Si on veut comprendre la dynamique fondamentale du récit spontané, il faut avoir une idée du fonctionnement de la narration dans sa situation originale, donc un modèle de la dynamique intrinsèque de la narration. Celle-ci concerne la relation du texte (et de l'information qu'il contient) à l'événement raconté (le 'référent' du texte) et à l'imagination épisodale (dynamique) du narrateur et de l'auditeur, donc à la signification du texte narratif pour les systèmes cognitifs des participants. Comme l'événement raconté et le système cognitif qui l'a perçu, mémorisé et mis en mots ont une organisation dynamique, ils se prêtent à une modélisation à l'aide de la théorie des systèmes dynamiques. Il s'agit donc d'expliquer comment une structure linguistique, le texte qui traditionnellement donnait l'impression d'être une entité statique aux parties discrètes (les mots, les phrases), peut servir d'intermédiaire, de code pour un contenu dynamique. Ce problème central de toute analyse narrative implique les questions suivantes :

A. Quel est le rapport entre la structure et la dynamique de l'événement vécu et les structures lexicales, syntaxiques, textuelles qui apparaissent dans le texte?

B. Comment se reflète l'activité du narrateur, son point de vue, son intérêt, son évaluation dans l'organisation du récit?

Ces trois questions introduisent deux niveaux:

Le niveau référentiel : les événements et actions présentés dans le récit.

Le niveau évaluatif: la perspective choisie et imposée par le narrateur (en vue d'une évaluation par l'auditeur).

Cette dynamique à deux niveaux est valable pour le récit spontané utilisé en conversation où le narrateur présente un vécu personnel. Dès que le locuteur reprend une narration ou qu'il fait partie d'une chaîne de locuteurs qui reprennent la même histoire, il entre dans une dynamique de la réorganisation que nous appellerons une dynamique secondaire. Enfin un contenu peut acquérir une symbolisation supplémentaire, représenter des valeurs culturelles, religieuses ou mythiques. Dans ce cas, un narrateur peut simplement reproduire cet acquis culturel.

Dans une perspective holistique, le texte narratif a une gestalt (forme), c'est-à-dire qu'il passe d'un début à une fin marquées, qu'il a des contours temporels et qu'il contient une sorte de point culminant (climax) entre les deux, et utilise un ensemble limité d'actants centraux. Dans une perspective componentielle, on peut se demander :

1) Quels sont les segments de l'événement qui donnent lieu à une verbalisation dans le texte narratif?

2) Y a-t-il un vocabulaire minimal de schémas actantiels qui réapparaissent dans l'organisation de la narration?

3) Quelle est la syntaxe (la sémantique, la pragmatique) pour la composition d'un texte narratif à partir d'une telle segmentation ? 
Depuis l'œuvre de Propp, de Greimas et autres, des formules statiques (suite de catégories narratives) ont été proposées. Sous l'aspect dynamique nous allons plutôt considérer des évolutions prototypiques et nous parlerons, après René Thom, d'une morphogenèse de la gestalt narrative.

Si la narration contient deux éléments narratifs, c'est-à-dire au moins deux centres prédicatifs (par exemple dans des verbes finis), la question de la relation de ces deux centres prédicatifs dans la structure narrative se pose. Beaucoup de paires complémentaires de verbes suffisent à cette condition. La littérature narratologique nous propose par exemple: manque/complétion du manque, réunion/séparation (du sujet et de l'objet), confrontation du sujet et de l'objet et vice versa, complication et résultat. Dans le cas de trois unités narratives, on peut intercaler une unité dans le champ bipolaire. Cela fait apparaître la fonction du climax (voir Labov et Waletzky, 1987). Quand le schéma tripartite est atteint, on peut procéder à des bifurcations secondaires. La complication (comme fonction holistique) peut 'émettre' la fonction appelée 'orientation'; le résultat peut émettre la fonction appelée 'coda'. Ensuite les pièces premières (la complication et le résultat) peuvent être séparées en épisodes. Le schéma morphogénétique est une réinterprétation génétique des structures arborescentes en théorie de la narration.

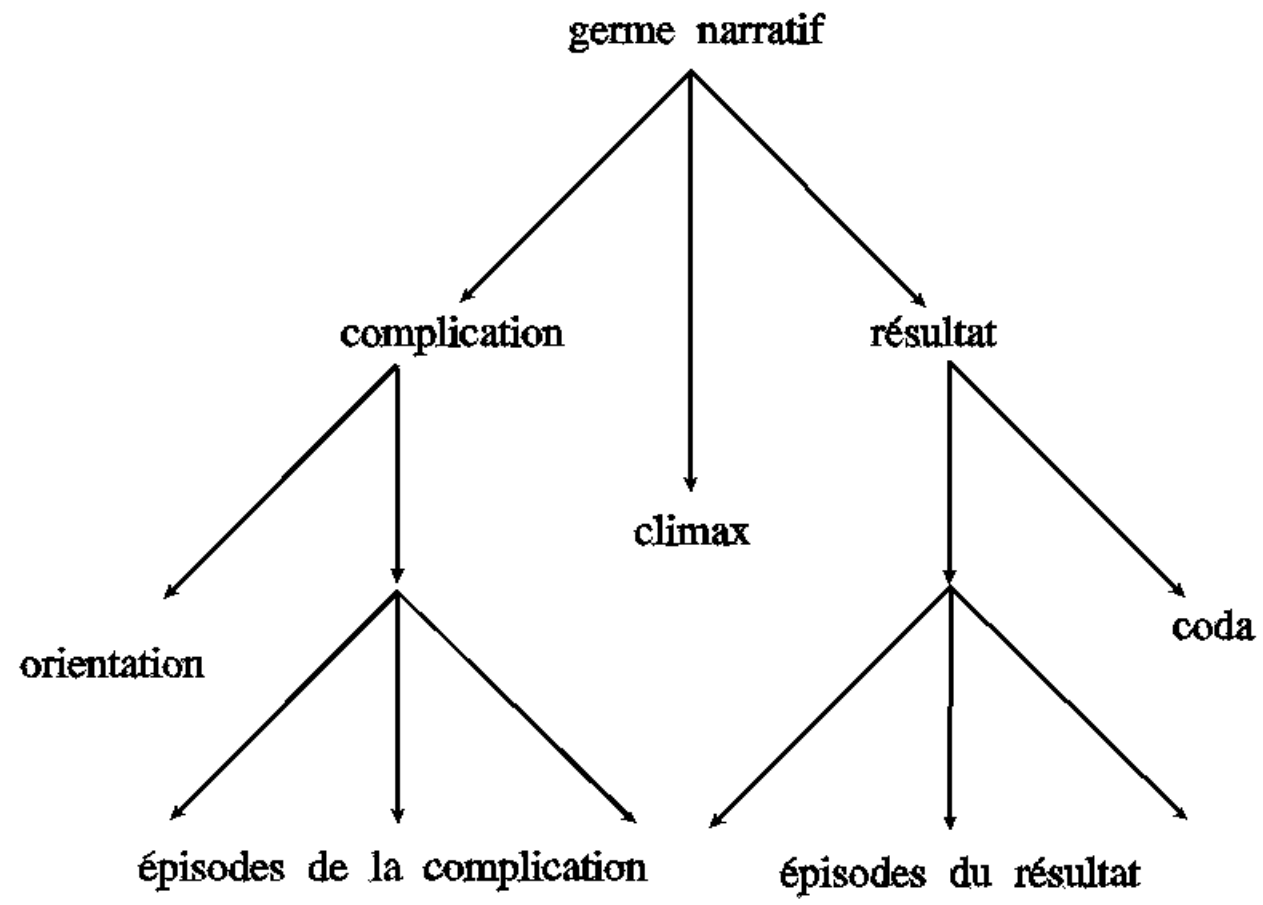

FIgURE 1. SCHÉMA MORPHOgÉNÉTIQUE dU TEXTE NARRATIF (RÉINTERPRÉTATION DU SCHÉMA DE LABOV ET WALETZKY (1987)

Ce schéma présente certaines asymétries :

- Les épisodes de la complication sont normalement plus élaborés, plus différenciés que ceux du résultat.

- Les constituants secondaires (l'orientation, le climax, la coda) possèdent des fonctions au-delà de la référence narrative qui dépendent de leur position dans la chaîne narrative.

Les cycles narratifs, résultat d'une morphogenèse catalytique (avec des boucles), sont rares dans les récits spontanés; la morphogenèse normale est donc acyclique ${ }^{3}$. Le 
caractère algorithmique du langage, mis en relief par les grammaires génératives, semble être le produit d'une réorganisation artificielle.

L'apparition du climax dans le champ bipolaire, constitué par les fonctions de complication et de résultat, peut recevoir une modélisation dynamique plus spécifique, que l'on retrouve également dans d'autres domaines. Il s'agit d'un comportement typique de certains systèmes dans l'environnement du point catastrophique (de la déstabilisation, du changement). La direction de cette dynamique dépend d'ailleurs d'une évaluation positive (le déroulement est arrêté, ralenti) ou négative (le déroulement est accéléré). Nous donnerons un modèle catastrophiste pour le déroulement freiné qui est typique des récits qui montrent le narrateur comme le héros, le vainqueur qui résout le problème. Dans le cas d'un aveu où le narrateur décrit un méfait ou un crime, on peut observer une transition très rapide de la complication au résultat.

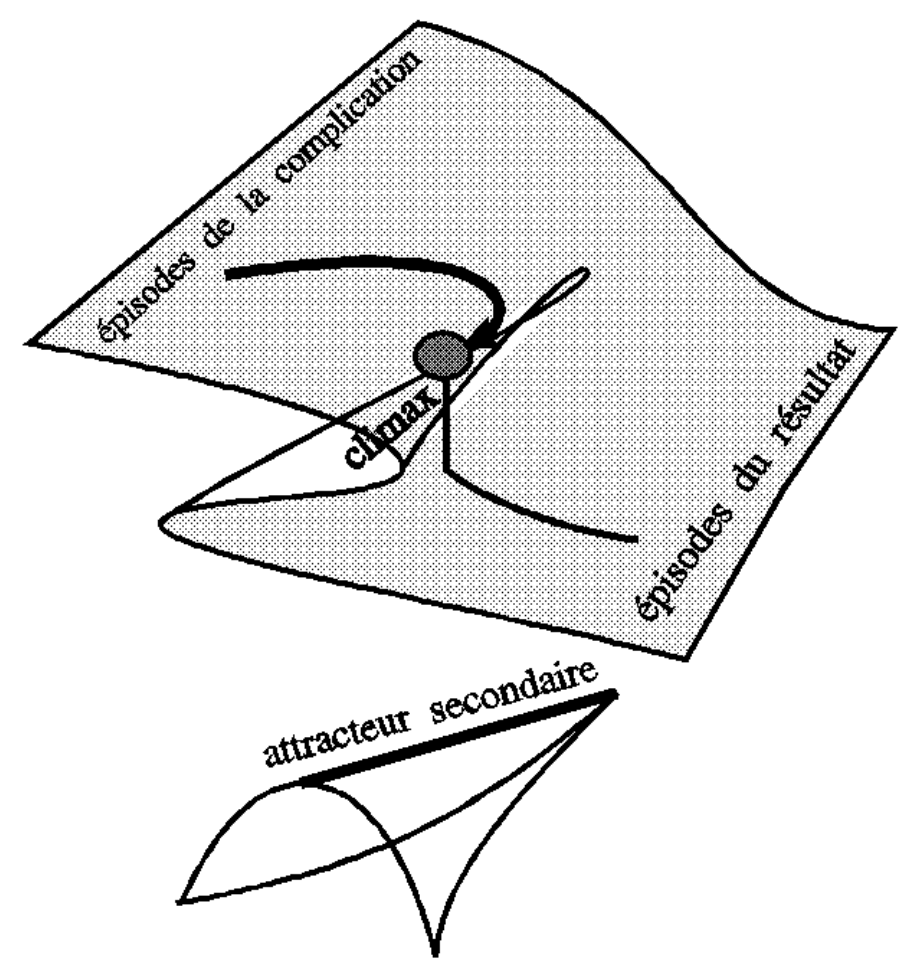

FIgURE 2. LA MORPHODYNAMIQUE dU PASSAgE À TRAVERS LE CLIMAX

La fonction évaluative ne concerne pas seulement le développement de l'événement dans son ensemble, elle peut aussi bien contribuer à l'élaboration de la complication et du résultat et des épisodes que ceux-ci contiennent. Dans ce dernier cas, la fonction évaluative est plutôt réalisée au niveau des choix lexicaux ou dans la construction des groupes nominaux et verbaux (par des adjectifs et des adverbes évaluatifs). En général, les modifications évaluatives s'appliquent à tous les niveaux linguistiques et ne concernent pas uniquement les structures textuelles et transphrastiques.

Les fonctions d'orientation et de coda sont, comme le climax, motivées par la fonction évaluative (pragmatique). Le point de départ est la bipolarité évaluative :

narrateur (l'origine pragmatique du récit) / auditeur (point de destination du récit) 
Le côté gauche de la polarité est fondamental étant donné que le narrateur est centré sur lui-même. Le côté droit correspond au fait que le narrateur doit 'indemniser' son auditeur pour avoir le droit à une séquence plus longue que l'unité minimale dans une dynamique conversationnelle (voir Sacks, Jefferson et Schegloff, 1974). À l'intérieur de ce mouvement dans le champ pragmatique, on repère d'abord la dominance du destinateur/auditeur (qui motive l'orientation) puis le retour à l'attracteur normal : le narrateur, sa perspective, son évaluation de l'événement (qui produit le climax et la coda). Entre les deux extrémités, le narrateur réagit à la polarité de l'événement (négative ou positive pour lui-même) soit en éliminant le climax (fuite devant une évaluation), soit en l'accentuant (recherche d'une évaluation).

Morphogenèse du conte populaire

Les contes populaires tels qu'ils nous sont présentés par les frères Grimm en Allemagne et par Perrault en France sont dans une certaine mesure le produit final d'une tradition. Dans une culture orale, ces contes étaient porteurs d'un message historique et culturel. Dans le stade de la transformation du conte oral en un objet quasi-littéraire, le texte a été remanié en utilisant les techniques littéraires et les normes esthétiques de l'époque.

Notre analyse du conte 'Rumpelstilzchen' part de la version donnée par Grimm et la compare ensuite à une version plus proche de la tradition orale. La séquence narrative centrale peut être décrite par un schéma de don, de contre-don, avec une promesse de contre-don et une trahison (un don non-volontaire). Le don est une catégorie anthropologique et sociale (voir l'œuvre de Marcel Mauss); il a aussi un côté cognitif et évolutionnaire. Pankseep et Biven (2012: 95) décrivent un système émotionnel archaïque de «sources cérébrales de l'anticipation dynamique, du désir, de l'euphorie et de toutes sortes de quêtes " dans leur Archeology of the Mind, qu'ils appellent "The SEEKING system ». Le don est la réponse sociale à cette prédisposition émotionnelle de l'homme (et d'autres primates et mammifères). Une schématisation du simple don (non réciproque) fut proposée en termes mathématiques par René Thom dans son « archétype du don ». La Figure 3 reprend cette schématisation (voir Wildgen, 1994 : $70)$.

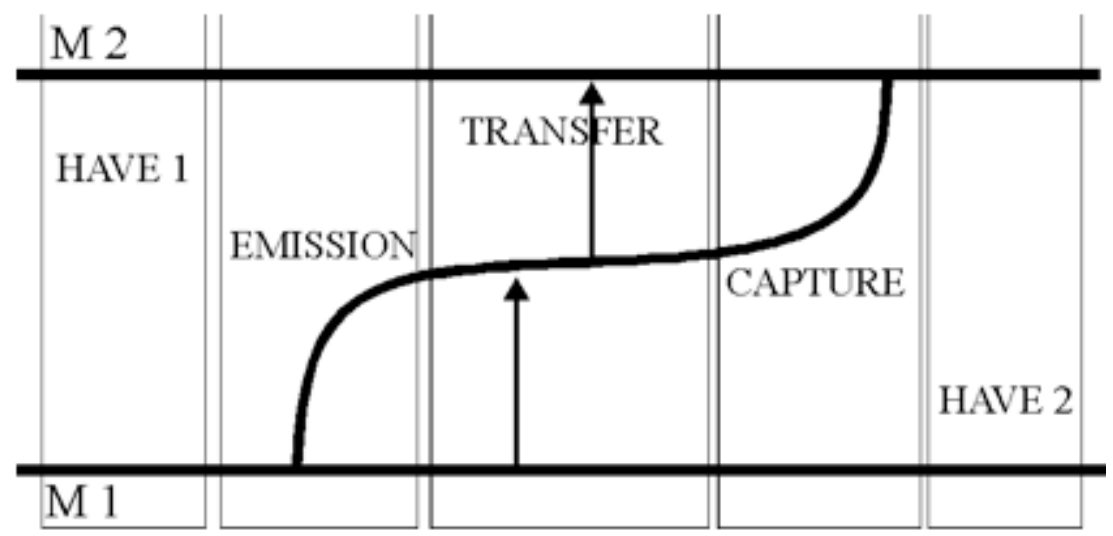

FIgURE 3. LE SCHÉMA DU dON EN SÉmANTIQUE CATASTROPHISTE

Nous utiliserons la schématisation proposée par Thom pour décrire de façon schématique le déroulement de la structure narrative d'un conte choisi dans l'œuvre des frères Grimm : 'Rumpelstilzchen'. 
Dans la phase de la complication, nous avons trois actants : la meunière (qui deviendra reine), le roi et le nain (ou lutin) 'Rumpelstilzchen'. La meunière est un actant intermédiaire dans le schéma du double don : elle reçoit pour donner. Tandis que le roi ne doit son don qu'à la fin de la série, la meunière fait un contre-don (pour l'or reçu) à chaque cycle. Au troisième tour, elle donne seulement la promesse d'un contre-don, le fruit de son mariage (qui est le contre-don du roi pour l'or reçu) : son nouveau-né. Le contre-don reste en suspens et définit le problème à résoudre dans la partie du récit avec la fonction 'résultat' (dans la terminologie de Labov).

Le résultat consiste lui aussi en trois phases avec un don intermédiaire. La reine essaie d'éviter le contre-don (le nouveau-né qu'elle doit au nain) et tâche de le remplacer par la résolution d'une énigme. Elle doit deviner son nom ('Rumpelstilzchen'). À deux reprises elle propose un nom, mais elle échoue. Enfin, le commissaire de la reine surprend le nain qui, en chantant, trahit son nom (il donne ainsi, sans le vouloir, la résolution de l'énigme). La reine, informée par le serviteur, peut alors sauver son enfant. Ce contenu peut être géométrisé dans le cadre d'un modèle dynamique du récit. Nous choisissons une représentation semi-technique et illustrative qui n'utilise que les schémas du don et du double don (voir Thom, 1983 et 1988).

\section{complication}

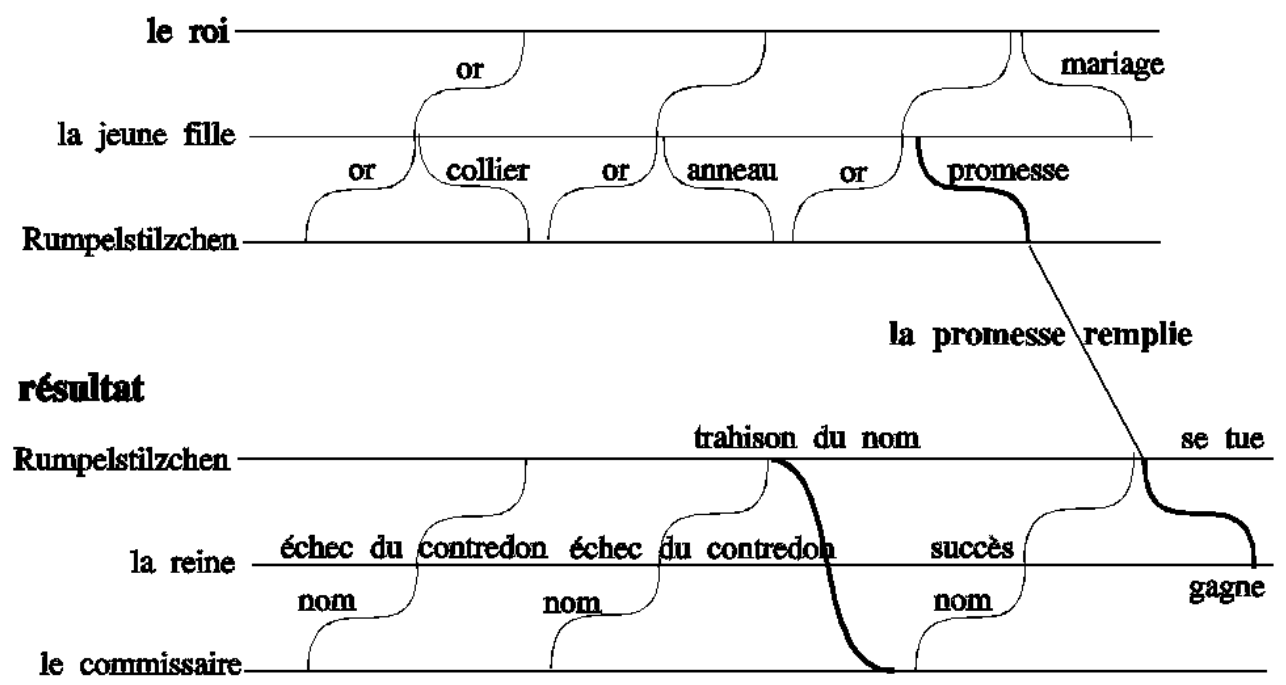

a rempli son devoir

Figure 4. Schématisation de la phase de la complication et du résultat

Dans une version plus ancienne et plus authentique du même conte, la jeune fille ne sait que filer de l'or et elle est très malheureuse du fait de cette 'inhabilité'. Le nain propose de lui envoyer un prince qui saura apprécier ce qu'elle fait et qui va l'épouser. En échange, il demande le nouveau-né. Tout se passe comme promis. Quand l'enfant naît, le nain consent à ce que la reine devine son nom au lieu de lui donner l'enfant. Le troisième jour, la servante observe le nain qui chante et qui, se croyant seul, trahit son nom. La reine devine le nom et sauve son enfant. En utilisant les mêmes techniques et conventions, nous pouvons en déduire une schématisation comparable à celle de la Figure 4. 
le prince apparaît

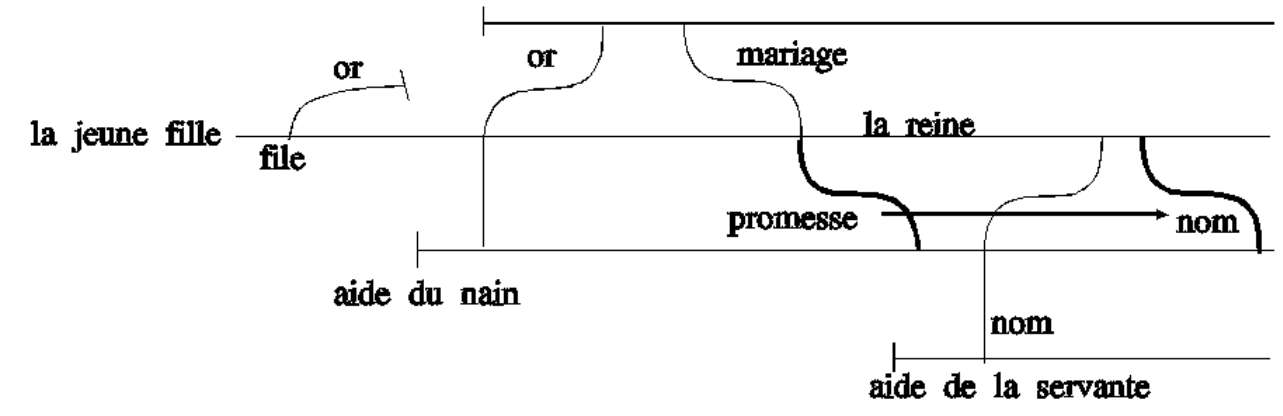

FIgURE 5. SCHÉMATISATION DE LA VERSION ORIgINALE

Dans cette version, le don et le contre-don ne concernent que la jeune fille et le prince. La relation entre le nain et la jeune fille a un caractère magique et se situe en dehors de la causalité naturelle. On voit bien que le double don, les symétries (pures et brisées), les rythmes ont été ajoutés dans la version publiée par Grimm. Il s'agit d'une réorganisation esthétique et formelle (elle concerne la forme et non la substance du contenu mythique). Les réorganisations littéraires (ou en général celles au-delà de la tradition orale) mettent en œuvre des techniques littéraires et esthétiques.

La narrativité dans la modalité visuelle

Cassirer a introduit une liste de formes symboliques qui déploient la capacité humaine pour le symbolique, la création et l'emploi des symboles/signes. Il a publié trois monographies sous le titre Philosophie des formes symboliques: le tome 1 sur le langage (1923), le tome 2 sur le mythe (1926) et le tome 3 sur la forme symbolique du savoir scientifique (1929). Cassirer met un relief la pluralité des formes symboliques qui coexistent depuis les débuts de la civilisation humaine et il essaie de montrer la spécificité de ces formes, leur délimitation, leur profil fonctionnel. La modalité visuelle est le fondement perceptif de la forme symbolique que nous trouvons dans l'art de la peinture, la sculpture et l'architecture. Le film muet ajoute le mouvement perçu et le film moderne ajoute les couleurs et le bruit, le langage, la musique. Je vais discuter ici la narrativité dans l'art de la peinture et dans le film moderne, surtout dans les films de la série des James Bond, l'agent 007.

Je voudrais d'abord considérer l'ordre vectoriel des versions de La Vierge à l'Enfant avec Sainte Anne de Léonard de Vinci. Le contenu narratif des tableaux et sculptures avec le titre "Sainte Anne trinitaire" renvoie au proto-évangile de Jacques. Il raconte l'enfance du Christ en partant de ses grands-parents. On y trouve aussi le récit de l'enfance de la Vierge Marie. Le tableau de Léonard montre la scène où Sainte Anne, la mère de Marie, tient Marie avec son enfant Jésus sur ses genoux. Je souhaiterais donner rapidement une idée de la structure vectorielle du tableau ${ }^{4}$. 


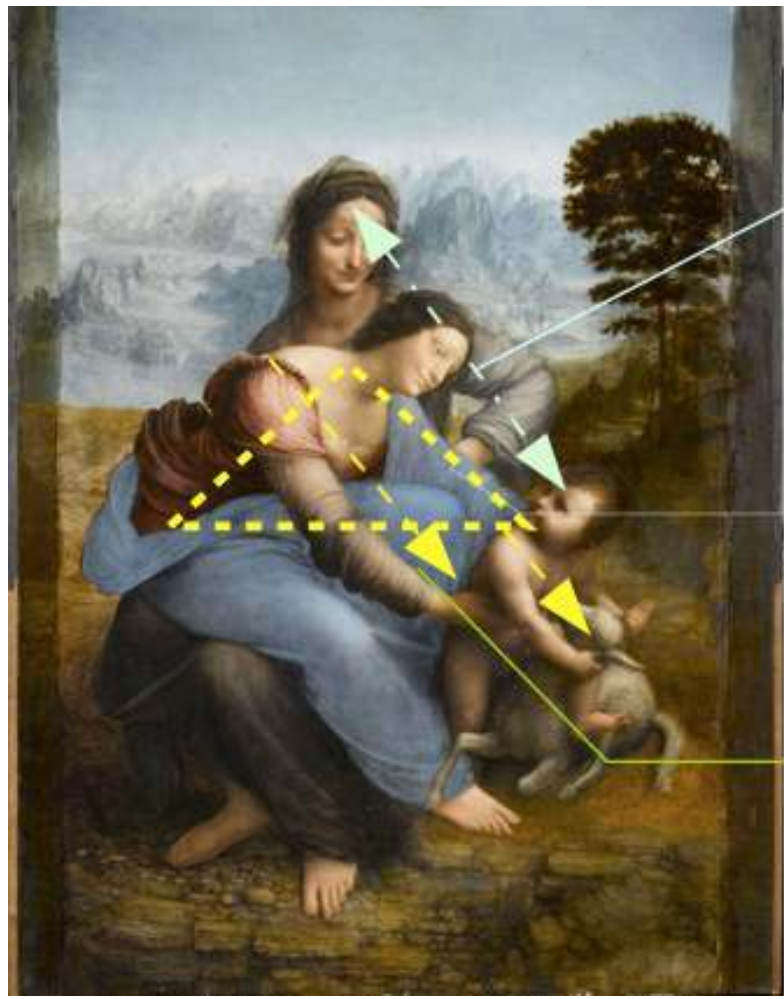

Ligne

principale du

regard en

parallèle avec

les lignes de

force des bras

L'équilibre

statique des

corps de St.

Anne et Marie

Lignes de force

de Marie et de

Jésus

Figure 6. La Vierge à l'Enfant avec Sainte Anne, 1509/10 (Paris, Louvre) et les principaux vecteurs de force et de regard.

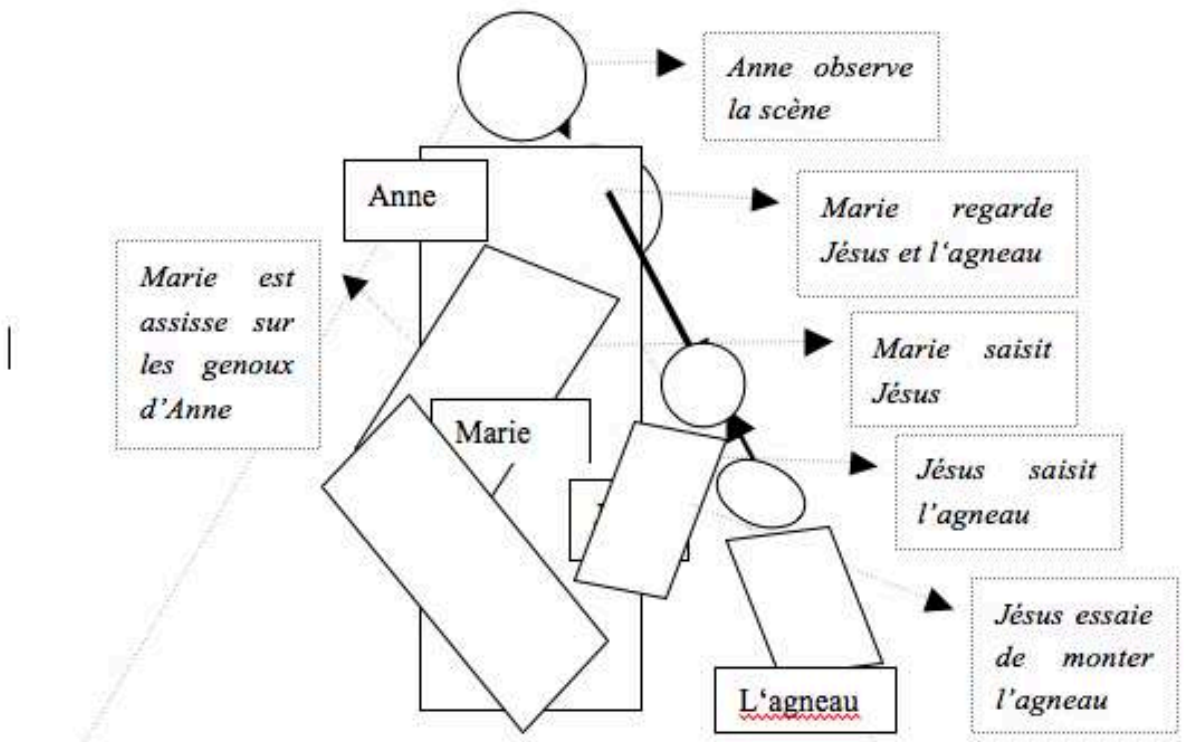

FigURE 7. SCHÉMA VECTORIEL DES CONTENUS VISIBLES DANS LE TABLEAU

D'une part on voit l'arrangement linéaire des regards d'Anne, de Marie, de Jésus et de l'agneau ; les deux derniers regardent vers le haut, les deux premières vers le bas. Les regards des deux groupes se croisent, ce qui démontre une opposition, un conflit. Contrairement aux esquisses préparatoires et au carton détaillé présenté par Léonardo 
à ses clients, on observe une simplification radicale de la structure vectorielle des regards (voir Wildgen, 2013a: 97-100). La structure visible fait reconnaitre trois types de situations/événements :

Marie est assise sur les genoux d'Anne,

Marie saisit Jésus,

Jésus essaie de saisir l'agneau et de le monter.

Si le « lecteur » du tableau imagine une entrée : Marie vient voir sa mère avec son fils et elle prend place sur les genoux de sa mère tout en tenant son fils, et une sortie : Jésus va jouer avec l'agneau et Marie s'assoit à côté de sa mère pour lui raconter ce qui s'est passé, on arrive à un récit dont la scène centrale, pour ainsi dire le climax du récit est présenté dans le tableau. Tout comme le récit parlé ou écrit qui ne représente qu'une lecture des actions (et guère leur déplacement spatial et temporel) le tableau permet une lecture qui décompose le tableau en sous-scènes visibles et les met en relation tout en produisant un "texte » visuel. Il serait faux de déduire de cette analyse que le tableau a une structure propositionnelle. Son contenu peut être transformé en un contenu propositionnel, si l'observateur verbalise sa lecture, mais il est présenté à l'aide d'un code pictural (visuel) qui reste dans la modalité visuelle tout en mobilisant un savoir plus général qui peut, entre autres, faire référence à un texte (par exemple à la lecture du proto-évangile de Jacques).

L'ordre géométrique (statique) n'a guère suffit à Léonard, qui a introduit un ordre vectoriel basé sur les mouvements et contre-mouvements du corps, l'équilibre de ces «torsions » et qui a pris en considération la direction des regards et des gestes. L'ordre vectoriel a pourtant une complexité restreinte: un, deux, trois corps en relation forment le champ accessible (voir la valence des verbes pour une restriction pareille). Le tableau peut être comparé à un récit résumé dans une phrase. Pour un récit de plusieurs phrases qui se suivent, il faudrait considérer une suite d'images, par exemple la série des xylographies de Dürer au sujet de la Passion du Christ ou, dans l'ère moderne, les bandes dessinées (voir Wildgen, 2013a : chapitre 7.2).

Le récit filmique

La photographie et le film sont des développements culturels récents. Le film était d'abord un «moyen de reproduction » (Malraux, 1947), et ce n'est que vers 1910-1915 qu'il a développé « un ensemble de signifiants spécifiques » (voir Metz, 1968:97) qui furent inspirés par le roman ou le théâtre et formèrent un «langage cinématographique »(ibidem: 96 ; Metz renvoie à Méliès, Porter et Griffith). Le « septième art » utilise les nouvelles techniques de l'image en mouvement, auxquelles il ajoutera bientôt la parole et le son. Dans le contexte des formes symboliques, l'aspect visuel des rituels, de la danse, du théâtre est élaboré par les techniques cinématographiques. Le cinéma hérite la pratique de la mise en espace et du mouvement du corps humain dans la sculpture et surtout dans le théâtre, et élabore leur diégèse et la prise en charge du narratif. Par cette évolution, le cinéma devient le nouveau noyau du narratif pour les masses.

Plusieurs genres de films mettent en avant les actions de force, la poursuite/l'évasion et la lutte/le combat. En termes d'une biologie de l'émotion (voir Pankseep et Biven, 2012) ils thématisent les ressources archaïques que les auteurs appellent RAGE et FEAR ( FUREUR » et « PEUR ») et dont ils analysent le support et les dynamiques neuronales. Je vais esquisser quelques aspects visuels et narratifs des films de James Bond, surtout à partir du film A Quantum of Solace (2008) 5 . 
Le film A Quantum of Solace montre déjà dans son ouverture une chasse sur les routes d'Italie (Lac de Garde, carrières de Carrare). Après la fuite du prisonnier à Sienne, Bond le poursuit sur les toits de la ville médiévale pendant que se déroule la fameuse course du Palio ${ }^{6}$. La chasse à l'homme traverse même l'arène de la course et elle finit sur l'échafaudage d'une grande salle à coupole. La chasse est un élément mythique traditionnel et elle trouve son climax dans la confrontation finale, lorsque Bond retrouve son arme et tire sur son adversaire, qui a lui aussi retrouvé son arme. Je veux montrer quelques aspects de la dynamique de cette chasse à l'homme.
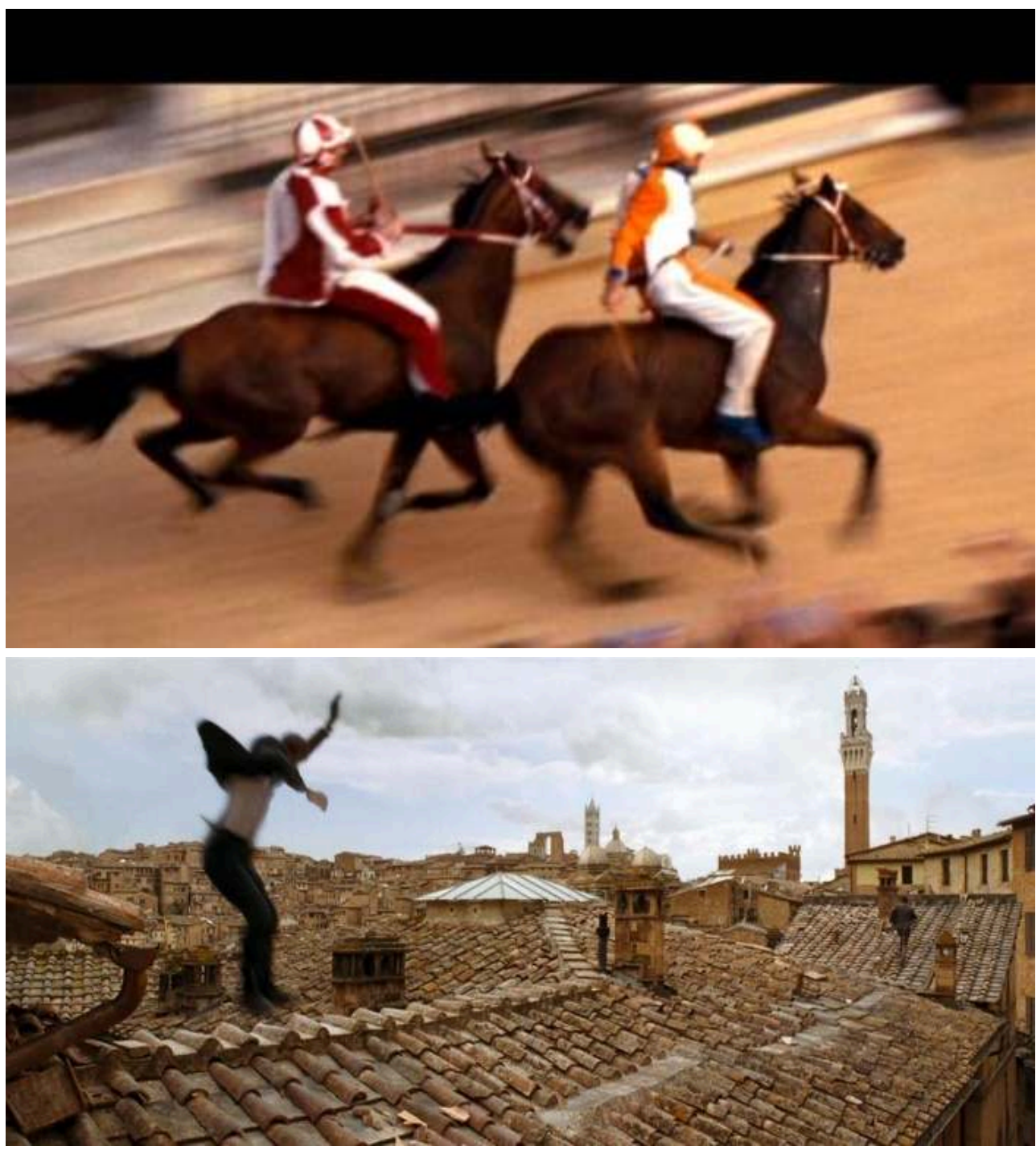

FIgURE 8. LA COURSE À SIENNE - BOND POURSUIT LE FUgITIF SUR LES TOITS DE SIENNE

Les deux processus sont indépendants mais se recoupent par moments : Bond et son adversaire traversent la foule - celle-ci regarde vers les toits où court Bond - la course est terminée et la confrontation finale met fin à la poursuite.

La chasse est linéaire, c'est-à-dire qu'elle suit un chemin; dans le cas de courses parallèles, celles-ci se recoupent dans une singularité. 


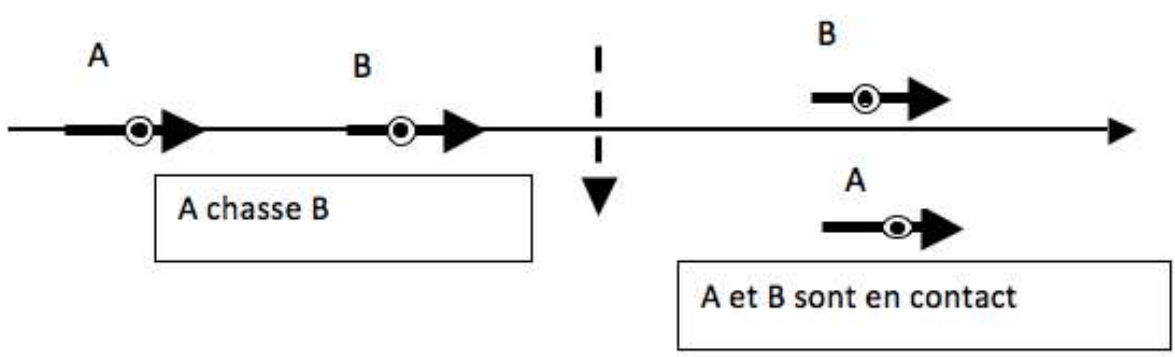

Figure 9. SCHÉmA de LA POURSUITE (LINÉAIRE) ET DE LA RENCONTRE

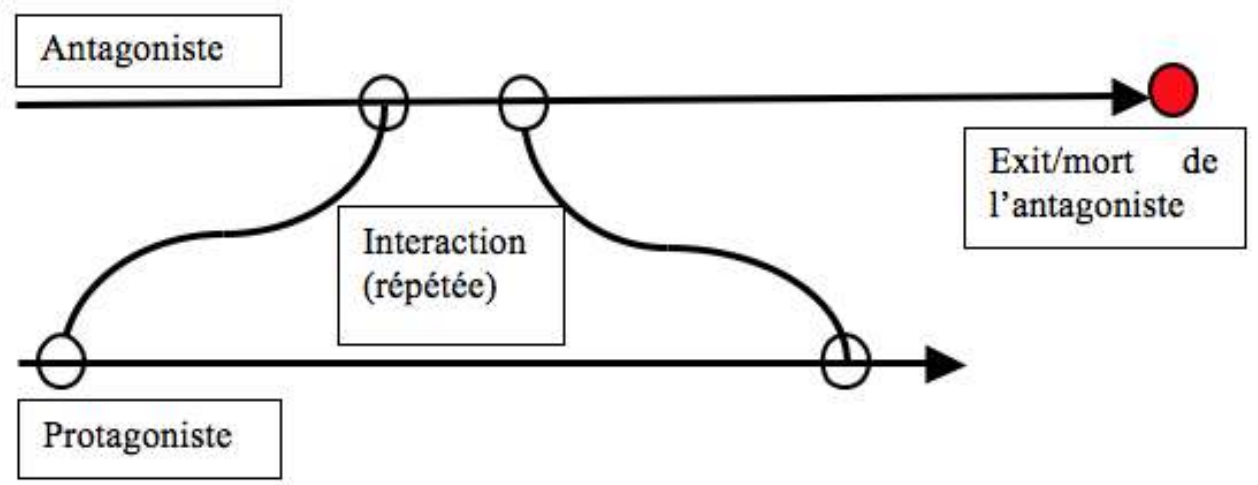

FigURE 10. SchémA de L'INTERACTION, DU CONFLIT (ÉCHANgE MUTUEL DE COUPS) ET EXIT

Dans la scène finale, la dynamique se complique. Bond et son adversaire interagissent surtout dans un plan horizontal et ils tombent dans un plan vertical (avec leurs armes). Les mouvements de poursuite se passent maintenant avec trois directions de liberté. Le combat sous la coupole (du cirque) connaît deux complications supplémentaires qui renvoient aux mouvements d'un pendule couplé et d'un pendule double (la situation est illustrée par le «screenshot » ci-dessous). ? 


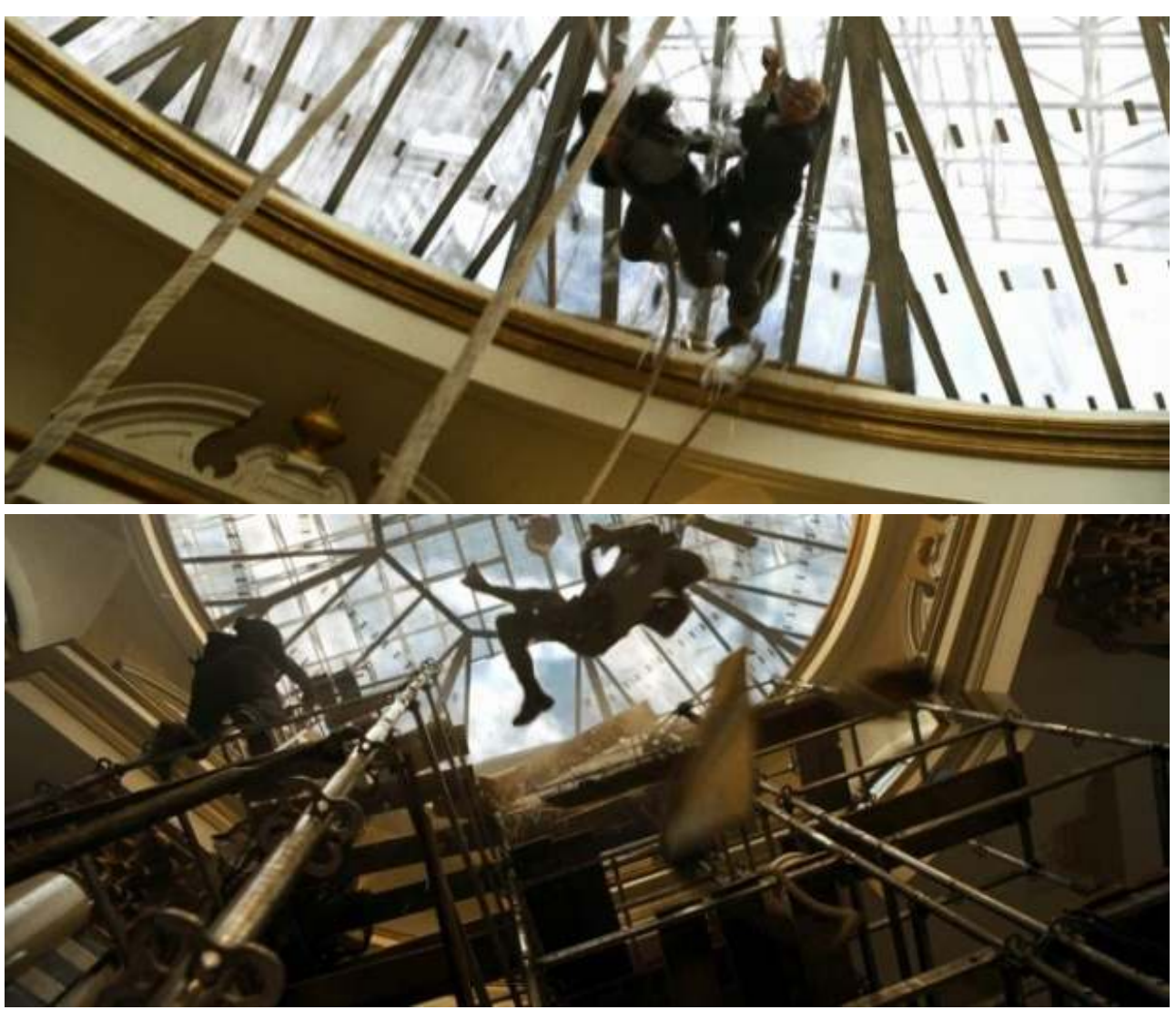

Figure 11. Le combat sous la coupole ; la dynamique des pendules couplés (à gauche) et du pendule double (à droite)

Le même film répète le schéma des chasses parallèles: (1) Bond chasse Green et son équipe dans les corridors et escaliers de l'opéra à Bregenz (Autriche). Bond finit par tuer un agent ennemi tandis que sur la scène de l'opéra où se joue La Traviata, l'action culmine dans un meurtre. (2) Bond se bat avec Green (qu'il quitte pour achever sa vengeance plus tard), tandis que son partenaire féminin Camille se bat avec le général qui avait tué sa famille, et finalement le tue (Bond et Green entendent le coup de revolver).

Il y a, au-delà des chasses qui mettent en rapport deux ou plusieurs personnes, une dynamique propre des lieux. Lors de la poursuite en voiture au début du film, les tunnels de la route le long du Lac de Garde, les camions qu'il s'agit de doubler, la mauvaise route de la carrière de Carrare forment des obstacles qui s'opposent aux participants de la chasse et qu'ils doivent surmonter, éviter ou dont ils peuvent profiter pour distancer l'antagoniste. Dans le combat final du film qui a lieu dans un hôtel au Pérou climatisé à l'aide de cellules d'hydrogène, l'explosion de l'hôtel et les corridors en flammes deviennent l'adversaire ultime et Bond protège et console Camille qui a achevé de se venger. 


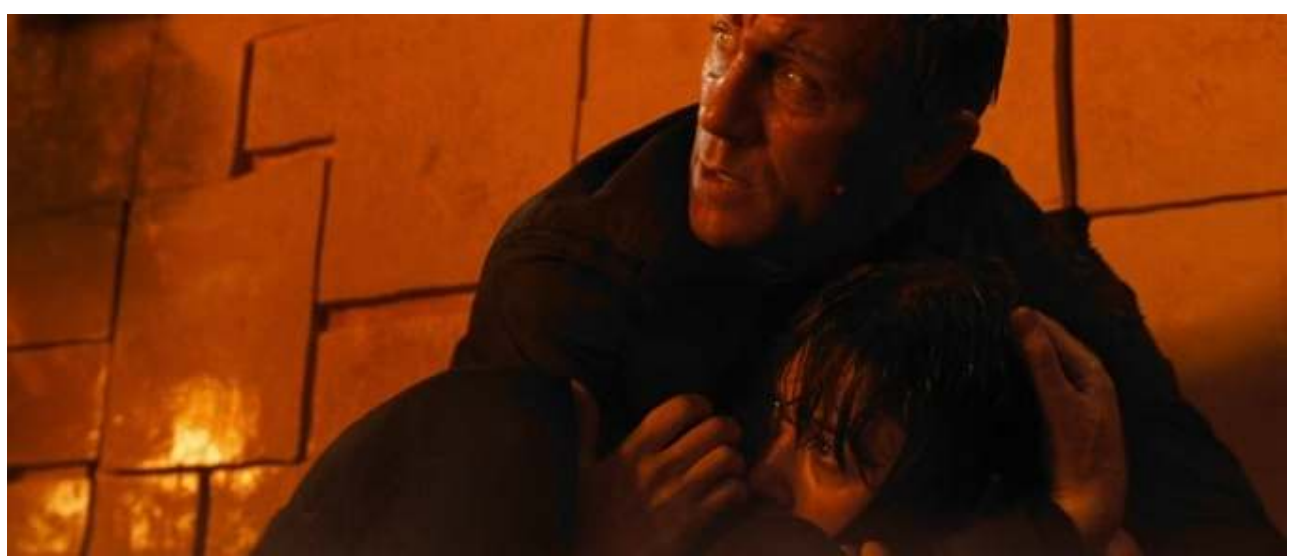

Figure 12. La consolation de l'héroïne par Bond (dans A Quantum of Solace, 2008)

Les scènes de chasse, de poursuite, la distinction des lieux sont un élément central dans les films de la série Bond. Dans le dernier film, Skyfall, le combat classique sur le toit d'un train en plein mouvement finit par la chute de Bond dans un abîme et, quasiment, son entrée dans le monde des morts. Les courants bouleversés d'un torrent finissent par le sauver, de façon presque surnaturelle.

Ces chasses n'épuisent pas l'univers narratif de la série Bond. Classiquement, on retrouve les jeux de casino (voir le film Casino Royale), les intrigues amoureuses, la corruption des agents ou contre-agents, etc. Si on compare ces films avec l'analyse des contes de fées chez Propp (1920/1970) on s'aperçoit que dans les deux cas un lexique assez réduit de schémas narratifs et évaluatifs est à la base du récit. Chez Bond il s'agit de sauver le monde ou l'humanité, au service de la reine et de la nation.

L'action, la chasse, le combat, les explosions et destructions forment le squelette dynamique du film, procurent un plaisir de cirque, basée sur la peur du spectateur et la capacité des hommes de reproduire quasi corporellement une action perçue dans l'imagination (voir le rôle des «minor-cells » et Rizzolatti et Arbib, 1998, pour la base neurologique de cet effet). Le thème de la chasse (et de l'amour) évoque des actions prototypiques et ancestrales. Le film ajoute un maximum d'illusion dynamique.

La narrativité en musique

Comme dans le cas de la sémiotique visuelle, il semble impératif de prendre un écart vis-à-vis d'une théorisation logique de la musique et d'éviter les analyses logicocentrées du langage dans le domaine de la musique. Koelsch (2012: 158) caractérise la musique en disant qu'elle renvoie pour l'essentiel à un type fondamental de « sens nonconceptuel» et négativement de sorte que les relations logiques: vrai/faux, connecteur, quantificateur, opérateur modal n'ont pas d'importance, c'est-à-dire qu'une « logique de la musique » n'existe pas. Colomb (1983) souligne qu'en raison de la prédominance de la fonction dénotative dans son langage, la complexité acoustique de celle-ci est réduite : il suffit que les segmentations et les classes sonores soient bien marquées de façon acoustique et auditive. En revanche, la musique déploie pleinement la texture du son ("sound texture ») en conformité avec la variété des dimensions et avec une complexité maximale ${ }^{8}$. De plus, les segmentations sont variables et peuvent être modifiées par l'interprète (encore plus radicalement par l'auditeur), c'est-à-dire que la musique n'est pas d'avance organisée en pièces discrètes comme le langage. C'est surtout le discours qui produit des limites fixes et intersubjectives (stables pour la majorité des auditeurs). Cela est particulièrement évident dans la musique indienne, ou 
dans le blues et le jazz. Pour la musique indienne (hindoustani et carnatique) Kühl (2007 : 178) écrit :

Chaque pas sur l'échelle peut être approché ou abandonné de façon spécifique. Il doit employer ses propres formes d'ornementation ou d'embellissement qui peut être un glissement d'une note à l'autre, une trémulation, un triolet, une suite plus élaborée de notes ou toute sorte de combinaisons de ces mesures.

Quant au Blues il remarque (ibid. : 179) :

un maître accompli du delta Blues comme B.B. King est capable de façonner sa musique à l'aide d'inflexions subtiles qui évoquent pour chaque blues un feeling unique.

L'auditeur en tant que participant au discours musical peut choisir librement dans la masse des impressions sonores présentées, tout comme le connaisseur d'art regarde le tableau à partir d'un ensemble d'impressions offertes. Son regard fait presque un parcours aléatoire, qui est différent pour chaque individu « lisant » l'image.

La sélection se fait de façon non linéaire - nous appréhendons ici un mot, là un rythme. - ce qui diffère de la sémantique textuelle, où on suppose que la compréhension se développe dans le temps et au niveau du texte qui se déploie. (ibid. : 476).

Par rapport aux signes visuels (par exemple dans le film) les signes musicaux ont une résolution temporelle très élevée. Spitzer (2009: 78) a noté que si notre résolution auditive était plus précise, nous entendrions même le bruit des molécules, ce qui ne serait pas agréable. Les cartes locales, que le cerveau produit sur la base de l'information visuelle, ont leur équivalent dans les cartes de fréquence du système auditif. Au-delà de cette différence au niveau sensoriel, les deux systèmes cérébraux fonctionnent d'une manière similaire. La langue diffère cependant par la primauté du mode de dénotation, qui se reflète également dans les différents processus cérébraux.

Sur le plan du discours, il est plus facile de comparer la musique avec le langage que sur le plan morphologique ou lexical. En particulier, on peut comparer : « des débuts et des fins bien développés, sous forme de pauses, de contours de la hauteur du ton, de l'accent dynamique et des structures rythmiques » (après Krumhansl, 1996 ; cité dans Koelsch, 2012 : 183). Le discours musical a lieu à différents niveaux :

Les créateurs de musique (compositeur, interprète improvisateur, auteur d'une chanson anonyme) entrent dans un discours avec d'autres créateurs et leurs œuvres, en reprenant des éléments pré-existants (citations ou variations) ou en créant une distance vis-à-vis de ces références.

Si la musique est présentée par un groupe (trio de blues, quatuor à cordes, orchestre ou chorale), un discours entre les musiciens a lieu, qui peut se développer spontanément dans l'exécution ou que le conducteur peut coordonner (par l'orchestre).

Le discours avec le public. L'auditeur peut répondre par des applaudissements, par l'expression de son désintérêt ou par déplaisir.

L'histoire de la musique montre la complexité historique du discours musical, son effet à long terme et la diversité des résultats. Je veux prendre comme exemple le genre du Blues (et ses conséquences dans le Jazz).

Le Blues remonte historiquement aux cris accompagnant le travail dans le delta du Mississippi, qui à leur tour ont leurs racines dans la tradition des chants d'appel et de 
réponse en Afrique de l'Ouest (Sénégal et autres) (voir Leroy 1963 : 90). Il existe donc un noyau discursif, une série d'énonciations qui sont transformées en musique. Du côté européen, nous avons les ballades qui contiennent une première énonciation (une phrase), le même contenu répété et une troisième ligne avec une information nouvelle comme fin de la strophe. Les strophes consécutives établissent une gestalt narrative, tandis que la strophe elle-même a plutôt un caractère performatif.

Toolan (2012) compare le texte littéraire, le film et la musique sous l'aspect de la narrativité. La suite des voix, chanteurs ou instruments fonctionne comme une suite d'actions liées à des personnes dans un récit. Celui-ci peut être raconté par une troisième personne ou mis en scène comme dans le film ou dans une pièce de musique. L'aspect du reportage indirect est mis en avant dans le récit littéraire, tandis que la mise en scène des actions et événements est davantage focalisée dans le film et dans la musique. Le récit littéraire est souvent déployé devant un arrière-plan fixe, un lieu qui est décrit, une institution, des personnages secondaires, etc. Ce phénomène existe aussi en musique où une ligne de basse (basso continuo) ou un thème qui revient sous plusieurs variations forment un tel arrière-plan. Il est certain que le « récit » musical est rarement descriptif ou référentiel au sens de personnes ou de situations très spécifiques, qu'on peut identifier dans le temps et dans l'espace. Mais cette exigence, qui devrait certainement être remplie par le protocole policier, par exemple, ne vaut guère pour les récits fictifs en littérature ou pour les contes populaires que nous avons discutés plus haut.

Quant aux centres neuronaux responsables de la compétence musicale d'un humain, on a d'une part trouvé un grand nombre de structures qui, lors d'une lésion par exemple, ont des conséquences pour la compétence musicale du patient. Avec le niveau de l'acculturation musicale et le degré de complexité de la musique, l'activité neuronale s'étend des centres associatifs auditifs A1, A2 vers les régions temporales, pariétales et frontales, pour impliquer enfin tout le cortex. En même temps les cerveaux d'un auditeur naïf et d'un musicien professionnel se distinguent foncièrement (voir la Fig. 22.2. dans Altenmüller, 2009 : 351).

En narratologie, on observe le rôle des matrices conventionnelles, qui garantissent la stabilité du récit et sa transmission sans grandes pertes (pour les matrices narratives, voir Nattiez 1983 : 1726). Cet aspect d'une conventionnalité stricte, qui sert de cadre pour une infinité de variations, est typique pour le blues et surtout pour les différents styles de Jazz qui ont évoluées aux États-Unis et plus tard en Europe.

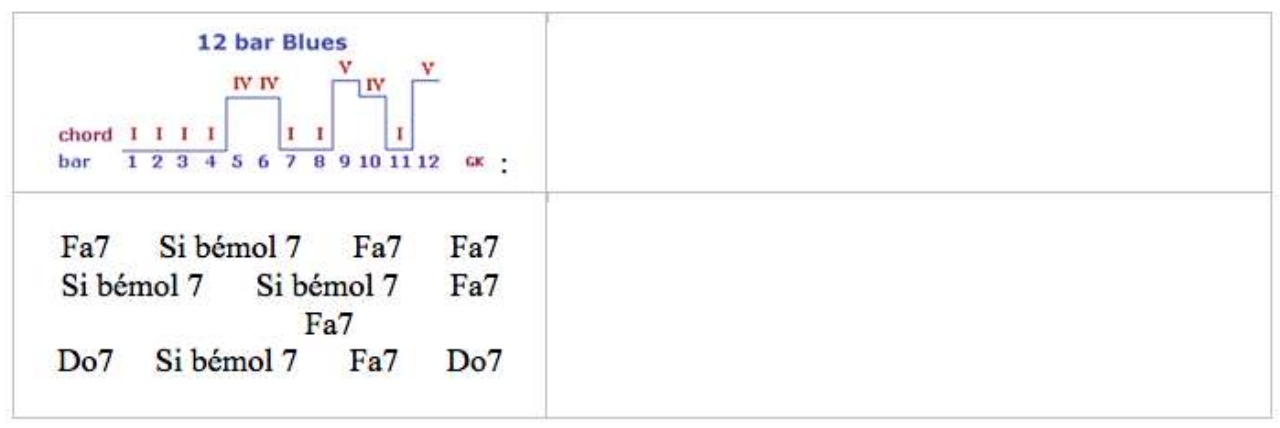

FIgURE 13. REPRÉSENTATION gRAPHIQUE DU SCHÉMA EN DOUZE TACTS ET SUITE d'ACCORDS DE BASE DANS UN EXEMPLE (BLUES EN FA)

Le système commence par la tonique (I), se déplace vers le deuxième centre, la sousdominante (IV), retourne à la tonique et va finalement vers le troisième centre, la 
dominante $(\mathrm{V})$ pour retourner à la tonique. De ce schéma, il y a d'innombrables variations. En outre, les instruments et les musiciens peuvent prendre leurs tours. En dépit des variations multiples, un motif discursif reconnaissable est maintenu. Le Jazz a développé ces caractéristiques: en particulier, dans l'improvisation et dans le déploiement du potentiel musical des nouveaux instruments (ajoutés au trio du blues: banjo, violon, percussion) il a élargi l'espace musical.

Conclusions

Nos analyses de la narrativité visuelle dans le film et musicale dans le Blues et la comparaison avec les résultats de l'analyse linguistique laissent apparaître une tendance générale: les formes symboliques ont une base commune, c'est-à-dire la capacité symbolique de l'homme. Comme les organes sensoriels et moteurs, auxquels elles renvoient, et les matériaux qu'elles utilisent sont différents, les formes symboliques divergent dans leurs manifestations concrètes et au cours du déploiement culturel de la capacité symbolique. Il semble que le langage ait, le premier, établi un inventaire de formes et une syntaxe de composition (au niveau morphologique et/ou syntaxique) de caractère algorithmique, donc répété à plusieurs niveaux. Les autres formes symboliques utilisent le même type d'accès à la complexité dès que l'évolution culturelle le permet ou le sollicite. C'est donc au stade de l'élaboration que des structures parallèles apparaissent (avec un langage visuel, filmique, musical). Une sémiotique visuelle et musicale doit considérer aussi bien la différence foncière (liée au corps humain et aux substrats de l'expression des formes) que les déploiements culturels qui introduisent un nombre de similarités dues aux exigences d'une communication complexe et stable dans une société de masse.

\section{BIBLIOGRAPHY}

Altenmüller, Eckart O., 2009. « How Many Music Centres are in the Brain ? », in Peretz et Zatorre 2009 (chapitre 22).

Cassirer, E., 1923-1929, Philosophie der symbolischen Formen : Die Sprache (1923), Das mythische Denken (1925), Phänomenologie der Erkenntnis (1929), Hamburg, Meiner 2000-2002.

Colomb, Harai, 1983. « Function-Reversal of Similar Subsystems in Different Auditory-Temporal Systems of Communication. The Role of Pitch and Timbre in Music and Language ", in T. Borbé (Hg.), Semiotics Unfolding, III, Berlin : Mouton, 1643-1646.

Greimas, A.J., 1966. Sémantique structurale. Recherche de méthode, Larousse, Paris.

Koelsch, Stefan, 2012. Brain \& Music, Wiley-Blackwell, Chichester.

Krumhansl, C.L. , 1996. « A Perceptual Analysis of Mozart's Piano Sonata K. 282 : Segmentation, Tension, and Musical Ideas », in Music Perception 13 : 401-432.

Kühl, Ole, 2007. Musical Semantics, Lang, Bern.

Labov, W. et J. Waletzky, 1967. « Narrative Analysis : Oral Versions of Personal Experience », in J. Helm (éd.), Essays on the Verbal and Visual Arts, Seattle : 12-44. 
LeRoi, Jones, 1963. Blues People. Schwarze und ihre Musik im weißen Amerika. Darmstadt : Melzer.

Malraux, André, 1947/1965. Le Musée Imaginaire, Gallimard, Paris (édition de 1965).

Mauss, Marcel, 2013. Sociologie et anthropologie. Introduction par Claude Lévi-Strauss, Quadrige, Puf, Paris.

Metz, Christian, 1968. Essais sur la signification au cinéma, Klincksieck, Paris.

Nattiez, Jean-Jacques, 1983. « A propos de Schoenberg : Les problèmes de la construction du modèle poétique en sémiologie musicale », in T. Borbé (Hg.), Semiotics Unfolding, III, Berlin : Mouton, 1717-1731.

Panksepp, Jaak et Biven, Lucy, 2012. The Archeology of Mind. Neuroevolutionary Origins of Human Emotion, Norton, New York.

Peretz, Isabelle et Zatorre, Robert J. (éds.), 2009. The Cognitive Neuroscience of Music, Oxford U.P., Oxford.

Propp, Vladimir, 1928/1970. Morphologie du conte, Seuil, Paris, 1970 (traduction du texte russe de 1969, édition corrigée de Propp, 1928).

Rizzolatti, Giacomo et A. Arbib Michael, 1998. « Language Within Our Grasp », in Trends in Neuroscience, 21(5) : 188-194.

Sacks, H., G. Jefferson et Schegloff, E., 1974. « A Simplest Systematics for the Organization of Turn-Taking for Conversation », in Language, $50: 696-735$.

Spitzer, Manfred, 2009. Musik im Kopf. Hören, Musizieren, Verstehen und Erleben im neuronalen Netzwerk. Schattauer, Stuttgart.

Stadler, M., et Wildgen, W., 1987. « Ordnungsbildung beim Verstehen und bei der Reproduktion von Texten ", in Siegener Periodicum zur internationalen empirischen Literaturwissenschaft (SPIEL), 6 : 101-144.

Toolan, Michael, 2011. « La narrativité musicale », in Cahiers de Narratologie [en ligne], n 21, 2011. Thom, René, 1974. Modèles mathématiques de la morphogenèse, Union Générale d'Editions, Paris (2e édition, Bourgois, Paris, 1980).

Thom, René, 1983. «Structures cycliques en sémiotique », in Actes Sémiotiques, 5 : 38-58.

Thom, René, 1988. Esquisse d'une sémiophysique. Physique aristotéliciene et théorie des catastrophes, Interéditions, Paris.

Wildgen, Wolfgang, 1994. Process, Image, and Meaning. A Realistic Model of the Meanings of Sentences and Narrative Texts, Benjamins, Amsterdam.

Wildgen, Wolfgang, 1999. De la grammaire au discours. Une approche morphodynamique, Lang, Bern.

Wildgen, Wolfgang, 2004. «Éléments narratifs et argumentatifs de l'«Ultime Cène» dans la tradition picturale du XIIe au XXe siècle », in Caliandro, Stefania et Anne Beyaert (eds.), Espaces perçus, territoires imagés en art, L'Harmattan, Paris.

Wildgen, Wolfgang, 2010. « Geometry and Dynamics in the Art of Leonardo da Vinci », in Cognitive Semiotics, 5 : 1-30.

Wildgen, Wolfgang, 2013a. Visuelle Semiotik. Die Entfaltung des Sichtbaren. Vom Höhlenbild zur modernen Stadt, Transcript, Bielefeld. 
Wildgen, Wolfgang, 2013b. « Erzählung und Action im James Bond-Film : Ein Quantum Trost », in Bateman, John, Kepser, Matthis et Kuhn, Markus (éd.), Film, Text, Kultur : Beiträge zur Textualität des Films, Schüren, Marburg : 321-345.

Fotos du film A Quantum of Solace : http://screenmusings.org/QuantumOfSolace/ index_19.htm\#2035

\section{NOTES}

1. Pour une modélisation en termes de morphodynamiques des niveaux de la grammaire au discours, cf. Wildgen (1999).

2. Pour la dynamique de la reproduction d'un texte ou d'une figure visuelle, voir Stadler et Wildgen (1987).

3. Voir pourtant Thom (1983) pour les structures cycliques en sémiotique.

4. Pour une analyse sémiotique de l'art de Léonard, voir Wildgen 2004 (français) et 2010 (anglais).

5. Voir pour une analyse détaillée du film A Quantum of Solace, Wildgen (2013b) (en allemand).

6. Elle a lieu deux fois par an, le 2 juillet et le 16 août. Le cercle de $300 \mathrm{~m}$ autour de la Piazza del Campo est parcouru trois fois.

7. Voir l'animation du pendule double sur ce site :

http://www.sciences.univ-nantes.fr/sites/genevieve_tulloue/Meca/Systemes/

pendule_double.php (lien consulté le 23 juillet 2015).

8. Colomb (1983: 1646) écrit : «La musique crée spontanément des unités discrètes à la base d'une échelle qui permet cette discrétisation sans y obliger. Ainsi en manipulant beaucoup d'éléments et de combinaisons dérivés d'une échelle simple et unifiée, la musique peut atteindre une complexité maximale de la texture tonale. ».

\section{ABSTRACTS}

Narrativity is basically linked to the dynamics of the events and actions which are told and it depends on the pragmatic context, mainly on the narrator and his audience, i.e. on the discursive setting. These dynamics ask for an adequate theoretical frame, e.g. in the format of dynamical system theory or vector analysis. Furthermore, narrativity can be manifested in different modalities. We shall present some specimens of analysis in the linguistic modality (spontaneous oral tales, folktales), in the visual modality (painting by Leonardo da Vinci, action movie in the series of Bond films), and in the musical modality (Blues music). The static analyses in the tradition of structuralism are replaced by morphodynamic schemes, by gesture and glance vectors, paths of pursuit and scenarios of conflict in action movies and eventually by harmonic sequences in the Blues. In this dynamic perspective, the notion of narrativity gains a different profile and it is freed from the preference for linguistic or logical categories.

La narrativité est foncièrement liée à la dynamique des événements et des actions représentés, et elle dépend du champ pragmatique narrateur/récepteur, c'est-à-dire du discours narratif. Cette dynamique demande une théorisation adéquate, par exemple au sein de la théorie des systèmes dynamiques ou de l'analyse vectorielle, et elle se manifeste dans des modalités différentes. Nous 
présentons des exemples d'analyse dans la modalité linguistique (récit oral spontané, conte populaire), dans la modalité visuelle (tableau de Léonard de Vinci, film d'action de la série James Bond) et dans la modalité musicale (Blues). Les relations statiques de l'analyse structurale sont remplacées par des schémas morpho-dynamiques, des vecteurs gestuels et du regard, des chemins de poursuite et des scénarios de conflit dans le film d'action et enfin des parcours harmoniques dans le Blues. Le concept de «narrativité » reçoit un profil différent dans cette perspective dynamique, et il est libéré de son orientation préférentielle linguistique et logique. 